\title{
Enhanced Power Within the Default Mode Network in Normal Subjects with Elevated Scores on an Egocentric Scale
}

\author{
Mark W.G. Collins ${ }^{\mathrm{a}}$ and Michael A. Persinger ${ }^{\mathrm{a}, \mathrm{b}, *}$ \\ ${ }^{a}$ Department of Psychology, Laurentian University, 935 Ramsey Lake Road, Sudbury, ON, P3E 2C6, Canada \\ ${ }^{b}$ Behavioural Neuroscience Program, Laurentian University, 935 Ramsey Lake Road, Sudbury, ON, P3E 2C6, Canada
}

\begin{abstract}
Integrated global power from the primary structures that composed the Default Mode Network (DMN) and from a random collection of other structures were measured by sLORETA (standardized low-resolution electromagnetic tomography) for young university volunteers who had completed an inventory that contained a subscale by which egocentricity has been inferred. Subjects who exhibited higher scores for egocentricity displayed significantly more power within the DMN structures relative to comparison areas. This was not observed for individuals whose egocentricity scores were lowest where the power differences between the DMN and comparison structures were not significant statistically. DMN power was greater in the right hemisphere than the left for men but greater in the left hemisphere than the right for women. The results are consistent with our operating metaphor that elevation of power or activity within the DMN is associated with greater affiliation with the self and its cognitive contents.
\end{abstract}

Keywords: Default mode network, Egocentricism, Gender differences, Psychometric Measurement, Quantitative electroencephalography, sLORETA.

\section{INTRODUCTION}

The default mode network (DMN) for intrinsic brain activity involves a neuroanatomically-based cluster of networks that are more likely to be activated when the conscious person is not focused upon the external environment. Fingelkurts and Finglekurts [1] reported a constellation of cortical areas that were operationally synchronized and persistent cross multiple experimental conditions. In general there is a network of pathways involved primarily with the medial prefrontal, anterior cingulate, posterior cingulate and inferior parietal regions which demonstrate increased power or energy utilization when the person is sitting awake with minimal exterogenic stimuli. The assumption is that the cerebral activity, in a manner similar to eliminating the input to an electroencephalographic system but increasing the gain, reveals the intrinsic activity of cerebral circuitry [2].

If structure dictates function then the greater involvement of these regions should determine the quality and themes of experiences that dominate the default mode. A blend of the somatosensory organization of the boundaries of the body, language-correlated imagery, and the free-associative but organized "stream of consciousness" mediated by the prefrontal regions $[3,4]$ should predominate. Because of the involvement of the anterior cingulate and the medial prefrontal regions, one would expect increased affect and

*Address correspondence to this author at the Department of Psychology, Laurentian University, 935 Ramsey Lake Road, Sudbury, ON, P3E 2C6, Canada; Tel: 01-705-675-4824; Fax: 01-705-671-3844;

E-mail: mpersinger@laurentian.ca "bonding" $[5,6]$ to this process. Stated metaphorically, the normal person should be emotionally bonded with his or her own thought processes and may find them as enjoyable as any low-level opioid-like process that is frequently associated with activation in this area [7]. We reasoned that if this constellation (the DMN) was that persistent and powerful, there should be significant positive associations between the electroencephalographic power of these structures and scores on psychometric tests by which "egocentrism" is inferred.

Research has indicated that an overlap between the areas activated during retrieval of autobiographical memory and the DMN exists. Ino et al. [8] found that the components of the DMN that were functionally related to autobiographical memory were observed in left-lateralized brain regions. This apparent contradiction with many studies that indicate that the active retrieval of autobiographical memory involves preferential activity of the right prefrontal region while left prefrontal activity emphasizes encoding [9] can be accommodated if one assumes that during engagement of the default mode retrieved autobiographical memories are reencoded.

Changes within the DMN has been reported for depressed patients who experience a marked increase in selfinspection and rumination whose themes are strongly dominated by negative and self-depreciating content that has been associated generally with persistent activation of mesiobasal temporal structures that have been disinhibited by hypometabolic prefrontal regions. In depressed patients the activity of the DMN does not decrease during tasks, as it does for normal individuals [10]. Although the comparison between obsession or repetition of negative thoughts 
Table 1. Items contained within the primary cluster by which "egocentricity" was inferred (egomain) and the additional items included in the original PPI cluster. The (-) indicates a positive contribution required a no response.

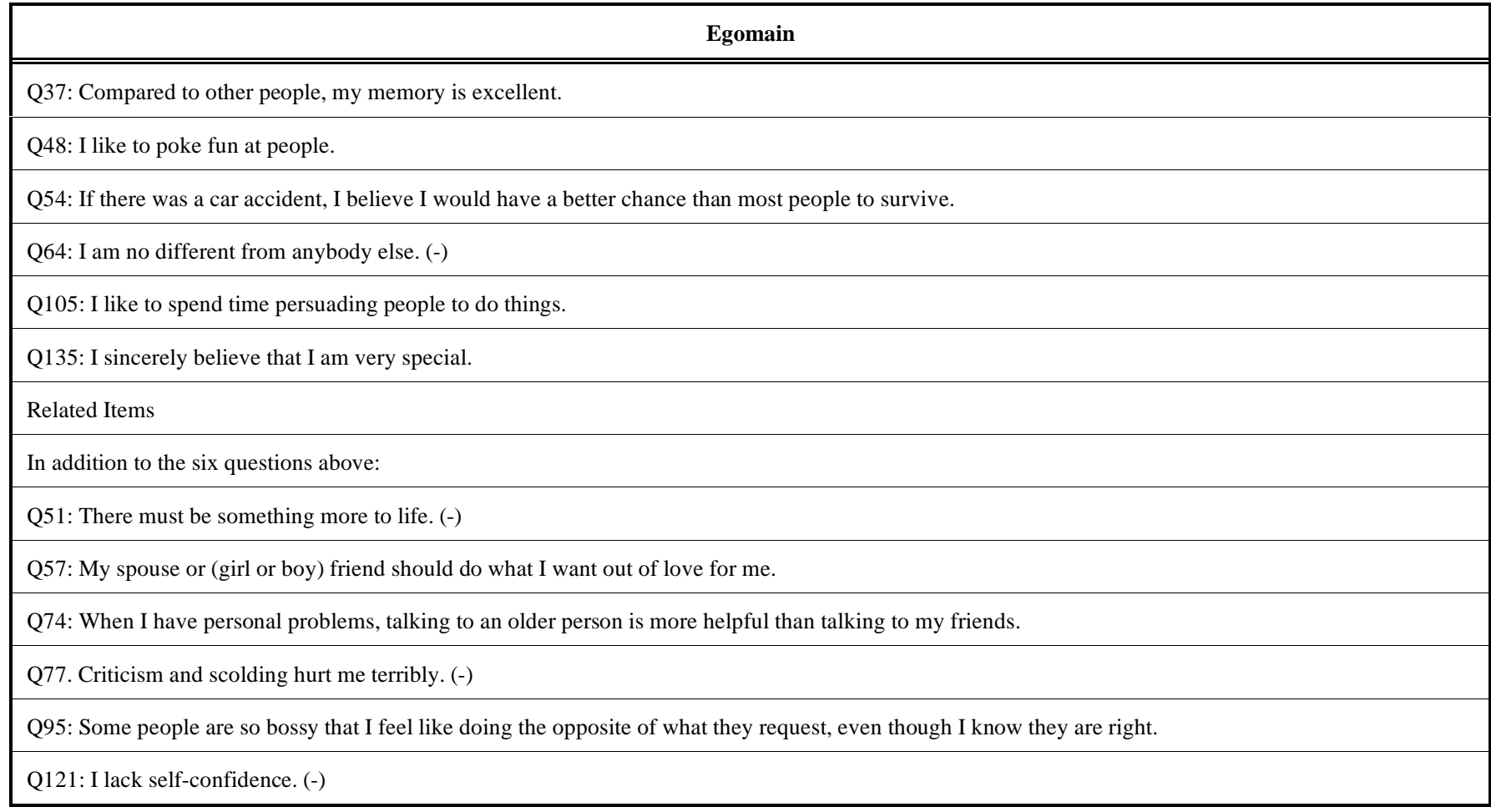

concerning the self (including self-destructive impulses) and addiction to the content of the ideation may be considered overinclusive, the similarities are striking. A similar pattern has been observed for certain types of dementia [11].

We hypothesized that if the DMN was that powerful it should be associated with increased psychometric indicators by which egocentricism has been indicated. Sheng et al. [12] found greater task-related deactivation in the medial prefrontal cortices for individuals who scored higher (equivalent to $\mathrm{r}=0.46$ ) on the Machiavellian Egocentricity scale that assesses narcissistic and ruthless attitudes during interpersonal functioning. In the present study we predicted that average university students who scored higher on an egocentric scale derived from the Personal Philosophy Inventory or PPI [13-17] should display more activation within the DMN as inferred by increased power measured by sLORETA or low resolution electromagnetic tomography during the default state, that is, relaxing and "day dreaming", within a quiet (echoic), darkened chamber.

\section{MATERIALS AND METHODOLOGY}

\section{Participants}

Sixty (60) volunteers had been recruited from the general university community for participation in several studies by different experimenters. Following approval by the university's ethics committee, the experimenters had asked for participants by delivering brief descriptions of their purposes which ranged from EEG correlates of meditation to various cognitive tasks. In the final analyses due to incomplete records there were 56 participants $(20$ men, 36 women). Their ages ranged from 19 to 28 years.

\section{The Psychometric Tool}

As a component of course participation all subjects had completed the 140 item Personal Philosophy Inventory (PPI) within the classroom within a few days to about four months before collection of electroencephalographic measures. The items that comprised the egocentric scale from the Personal Philosophy Inventory or PPI are shown in Table 1. The main scale (egomain) was composed of 6 items while the primary egocentric scale for the original cluster from the PPI was composed of 12 items. The six items were extracted following the original factor analyses. The PPI was initially developed as a psychometric indicator of sensitivity or "lability" within the temporal lobes. Construct validity and cross-validity for the clusters by which electrical lability within the temporal lobes has been inferred have been established [17].

In the tradition of specifying the psychometric properties of the tool, values were obtained from the 1,211 cases from our major data base. The average of the individual item correlations with the mean of the 6 items, derived from factor analyses of 12 items that displayed "face validity" as related to "egocentrism", that composed the egomain scale ranged from $\mathrm{r}=0.44$ to 0.55 . The Cronbach's alpha correlation for reliability within items was 0.43 . The means and standard deviations in the sample were $52(25) \%$ for the 509 men and $40(24) \%$ for the 702 women in the sample. The gender difference was statistically significant $\left[F(1,1209)=88.99, \mathrm{p}<0.001 ;\right.$ eta $\left.^{2}=7 \%\right]$. The individual correlation coefficients for each of the other 6 other items in Table 1 ranged between 0.08 and $0.58(\mathrm{M}=0.43)$ with the mean value for that cluster. The alpha correlation was 0.08 . 
A subset of the total population (120 men, 153) women who had been given the PPI and the Minnesota Multiphasic Personality Inventory over four successive years indicated that men's affirmative responses were $(M(S D)=49(24) \%$ while women's scores were $41(28) \%$. This increased score for the egomain cluster for men compared to the women was statistically significant $[\mathrm{F}(1,271)=5.13, \mathrm{p}<0.05]$. All of the means of the MMPI scaled (T) scores ranged between 46 and 60 for the three validity and 10 clinical scales. The egomain score was significantly correlated (all p <0.001) only with the following subscales of the MMPI: Depression (-0.27), Hypomania (0.32) and Social Introversion (-0.26).

\section{Measurements and Data Analyses}

EEG recordings were completed for each person as he or she sat for $30 \mathrm{~min}$ in a comfortable chair within a dark, quiet chamber that was also a Faraday cage. An ELECTO-cap international electrode system with $19 \mathrm{AgCl}$ sensors corresponding to the International 10-20 system was placed over the head and referenced to the ears (both linked) for collecting monopolar EEG data. Impendence under $10 \mathrm{k} \Omega$ was verified for each sensor (Fp1, Fp2, F7,F3, Fz, F4, F8, T3, C3, Cz,C4, T4,T5,P3,Pz,P4,T6,O1, and O2 after the "1020" system). The EEG cap was connected to a portable laptop outside the chamber and employed a Mitsar 201 amplifier system. WinEEG version 2.84.44 working in Microsoft Windows XP was used to collect the data.

The QEEG data were collected at $250 \mathrm{~Hz}$ with a notch filter of 50 to $60 \mathrm{~Hz}$. Eye blink and general muscle artifacts were removed. The primary data for each subject was an artifact-free $15 \mathrm{~s}$ increment during the initial eyes-closed baseline which was common to all experiments before other manipulations were instituted. The baseline conditions were taken within $10 \mathrm{~min}$ of verifying the sensor impedances. Source localization analysis was computed on the EEG data using Standardized Low Resolution Electromagnetic Tomography (sLORETA; [18]). sLORETA uses standardized current density to calculate intracerebral generators of cortical activity and has low resolution but ideally zero localization error in the presence of measurement and biological noise [18].

For clarification all of the sensors contributed to the Regions of Interest (ROI). Unlike a typical scalp recording in which the ROI would correspond to a surface location (e.g., T4/T6 corresponding to the ROI of the right temporal region), sLORETA software localizes activity based upon the relationship between sensors in the entire EEG profile and the identified voxels are mapped to a standard Talairach model of a human brain. ROIs were computed by selecting Talairach coordinates and a surrounding $5 \mathrm{~mm}$ radius. Three regions identified in literature as belonging to the DMN were selected: the Medial Prefrontal cortex (Broadman Area 10), the Posterior Cingulate cortex (BA23), and the Inferior Parietal cortex (BA34). Three ROIs not associated with the DMN were likewise extracted: the Primary Visual cortex (BA 17), Frontal Eye Fields (BA 8), and the Primary Auditory cortex (BA 41). Separate ROIs for both left and right hemispheres were extracted for each structure. Total spectral power was separated into the traditional EEG Bands of Delta (1-4 Hz), Theta (4-7.5 Hz), Alpha1 (7.5-10 Hz), Alpha2 (10-13 Hz), Beta1 (13-20 Hz), Beta2 (20-30 Hz) and Gamma $(30+\mathrm{Hz})$.

All data was imported into SPSS 19.0 for Windows 7. The 15-s, eyes-closed, artifact-free baseline EEG raw power segments were extracted from each individual. An overall DMN score was computed by first summing all band powers for each ROI, and then averaging the total activation scores of the three DMN ROIs for each hemisphere, which was likewise computed for the non-DMN structures. To limit the diluting effect of dichotomizing the scores below and above the mean, participants' scores were divided into low $(n=24$; $\mathrm{M}=42 \%)$ medium $(\mathrm{n}=18 ; \mathrm{M}=32 \% ; \mathrm{SD}=7 \%)$ or high $(\mathrm{n}=14$; $25 \%, \mathrm{SD}=10 \%$ ). Analyses of variance involving the activation scores for the DMN or non-DMN regions in the left and right hemisphere as a function of higher and lower scores for the egocentric scale and gender were completed. Post hoc analyses for interactions involved combinations of Tukey $(\mathrm{p}<.05)$ and correlated t-tests where appropriate. All analyses involved SPSS-16 PC software.

\section{RESULTS}

The results of this study supported our hypothesis that higher scores for six key items (egomain) from a psychometric inventory by which egocentricism is inferred would be associated with more activation within the structures that comprise the DMN compared to reference structures. Three-way analysis of variance involving between the two groups with the upper and the lower egocentric scores and the two within levels (DMN vs reference; left $v s$ right hemisphere) was completed. There was a significant interaction between the egocentric groupings and DMN vs reference structure activity $\left[\mathrm{F}(1,36)=6.76, \mathrm{p}<.01\right.$; eta $\left.^{2}=.16\right]$. The source of the interaction was due to (see Fig. 1) the greater activation levels within the DMN structures by the group who scored highest on the egocentric scale compared to those who score lower.

Except for the obvious differences in power between DMN and references structures $[\mathrm{F}(1,36)=46.22, \mathrm{p}<0.001]$ there were no other statistically significant effects. Scores for individual structures did not differ between the left to right hemispheres, with the exception of the Posterior Cingulate Cortex (a component of the DMN) with a main structure cluster effect $\left(\mathrm{F}_{(1,31)}=4.81, \mathrm{p}=0.036\right.$, partial eta $\left.{ }^{2}=0.13\right)$ and the Primary Auditory Cortex (a component of the reference structure) with an interaction between structure and Egocentrism $\left(\mathrm{F}_{(1,31)}=4.70, \mathrm{p}=0.038\right.$, partial eta $\left.{ }^{2}=0.13\right)$. Total activity means for each structure and DMN/Non-DMN averages can be seen in Table 2 .

As a control for endorsement of items, a 3-way ANOVA was employed for the two groups that formed the upper and lower third of the distribution of mean scores for the six (6) other items in Table 2. Except for the expected differences in the activation score between the DMN and reference structures $\left[(1,34)=19.00, \mathrm{p}<0.001 ; \mathrm{eta}^{2}=36 \%\right]$ there were no significant main effects or interactions between the two groups with the upper and lower scores for the other items in Table 2 for activation scores. 


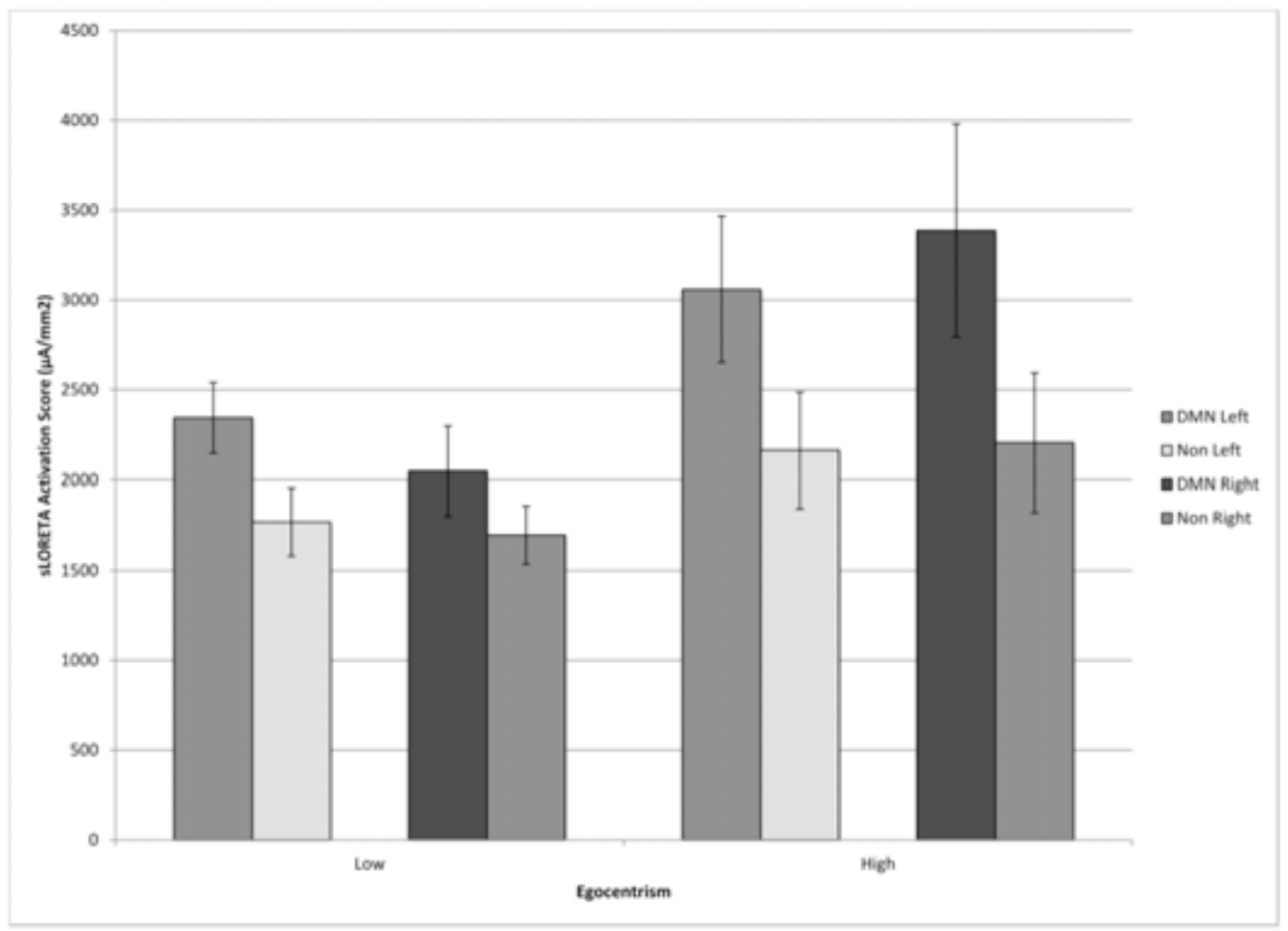

Fig. (1). sLORETA activation values $\left(\mu \mathrm{A}\right.$ per $\left.\mathrm{mm}^{2}\right)$ within structures that were included as the Default Mode Network (medial prefrontal, posterior cingulate, and inferior parietal areas) and reference (non-DMN) structures (frontal eye fields and primary auditory and visual cortices) in the left and right hemispheres for groups of individuals who scored lowest (1/3) or highest (1/3) on the egocentric scale. Vertical bars indicated standard errors of the mean.

Table 2. Means and Standard Deviations (in parentheses) for sLORETA Activation Scores (expressed as $\mu A$ per $^{2} m^{2}$ ) for $D_{M N}$ (DMN1, 2, and 3 refer to Brodmann Areas 10, 23, 34) and Non-DMN or reference (NON1, 2, and 3 refer to BA, 8, 17, 41) structures in the left and right hemisphere for subjects who scored within the "low" or "high" range defined by the psychometric "egocentrism" scale.

\begin{tabular}{|c|c|c|c|c|}
\hline & \multicolumn{2}{|c|}{ Low Egocentrism } & \multicolumn{2}{c|}{ High Egocentrism } \\
\hline & Left hemisphere & Right Hemisphere & Left Hemisphere & Right Hemisphere \\
\hline \hline DMN1 & $2855.29(1331.22)$ & $2931.51(1433.33)$ & $3470.96(1152.31)$ & $3534.89(1090.1)$ \\
\hline DMN2 & $1553.68(881.91)$ & $1589.43(895.52)$ & $2570.51(1253.41$ & $2586.24(1234.3)$ \\
\hline DMN3 & $2545.8(1694.76)$ & $2535.92(1859.35)$ & $4327.65(811.03)$ & $4090.68(719.46)$ \\
\hline DMN Mean & $2318.26(1302.63)$ & $2352.29(1396.07)$ & $3456.37(1072.25)$ & $3403.94(1014.62)$ \\
\hline NON1 & $1761.38(1852.35)$ & $1741.27(2144.15)$ & $1762.66(765.31)$ & $1864.32(908.93)$ \\
\hline NON2 & $2089.88(1943.09)$ & $2160.61(1951.95)$ & $3450.54(3026.5)$ & $3546.53(3130.62)$ \\
\hline NON3 & $1602.73(2964.04)$ & $1729.51(3185.28)$ & $2308.63(1505.21)$ & $2204.02(1970.13)$ \\
\hline NON Mean & $1817.99(2253.16)$ & $1877.13(2427.13)$ & $2507.28(1765.67)$ & $2538.29(2003.23)$ \\
\hline
\end{tabular}

One of the unexpected results was the large gender effect with respect to the hemisphericity of the power of DMN activity independent of egocentric scores. Three way analysis of variance as a function of gender, hemisphere, and DMN vs reference clusters demonstrated a statistically significant three way interaction $[F(1,52)=7.27, p<0.01$; eta $\left.^{2}=12 \%\right]$. Except for the main activity differences between the DMN and reference clusters there were no other statistically significant effects. The post hoc analyses (Tukey's, p <0.05) reiterated what is shown in Fig. (2). For men the DMN brain structures showed higher activity in the right hemisphere compared to the left while for women the DMN cluster showed higher activity in the left hemisphere compared to the right. 


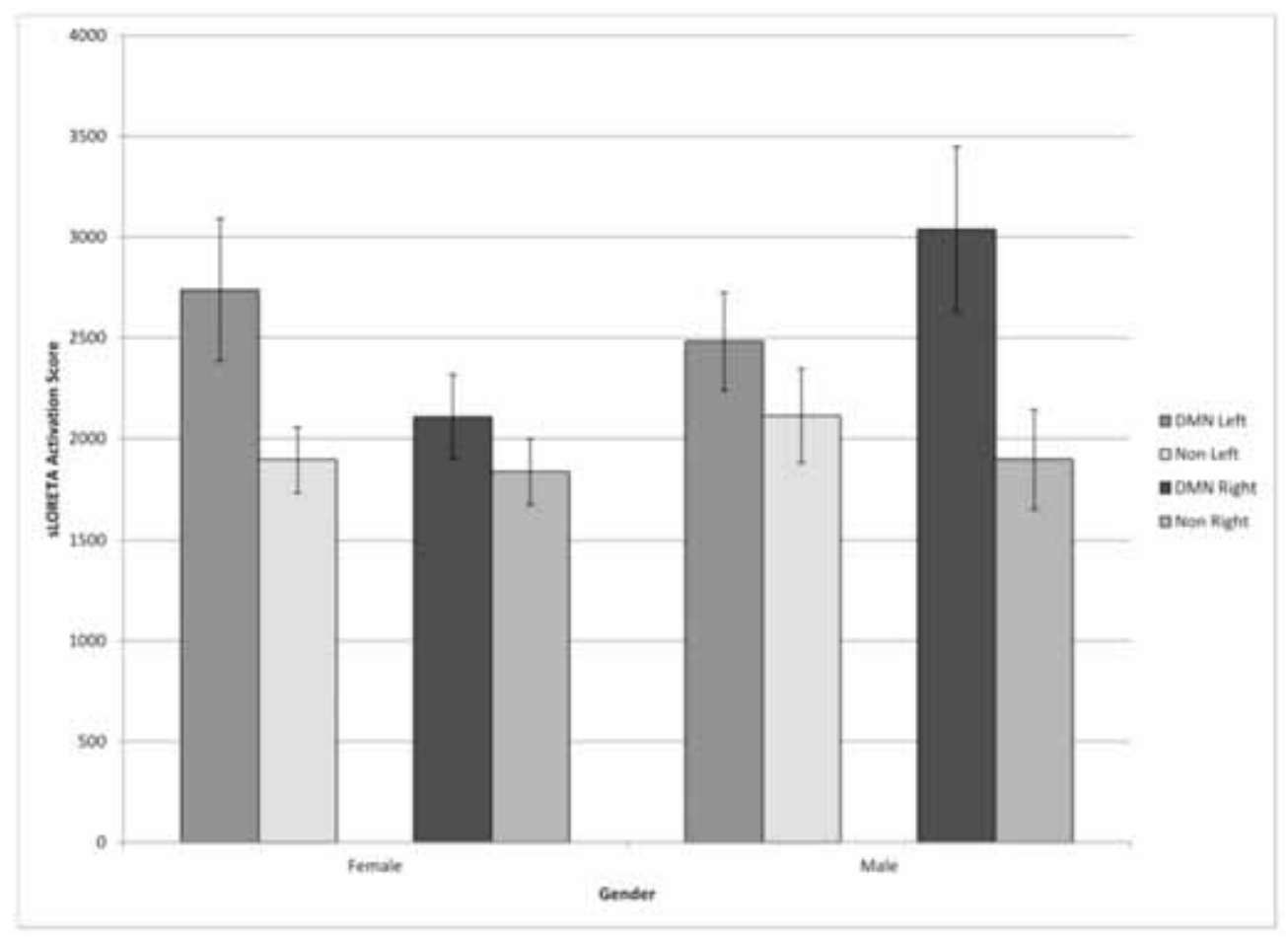

Fig. (2). sLORETA activation scores within the structures included as the DMN or reference structures in the right or left hemispheres for men and women. Vertical bars indicate standard errors of the mean.

\section{DISCUSSION}

The results of this study support the hypothesis that individuals who display elevated activity within cerebral structures associated with the classic components of the DMN are also more likely to score elevated scores on collections of items by which egocentricism is inferred. In this instance we are assuming the egocentricism reflects the propensity for the person to more confidently endorse his or her personal experiences as valid or "true" compared to people with lower scores. This pattern would also be congruent with our general model that self-appreciation or "self-bonding" to one's own thoughts may be a consequence of the function of the structures involved with the DMN as well as its persistent occurrence when external stimuli are minimized or no act upon the environment is required.

Six items from the same inventory that were initially included within the general "egocentric" scale but were found by factor analyses to be involved with a separate shared source of variance $[13,16]$ were not significantly associated with differential activation of the DMN structures compared to reference structures. The absence of the effect suggests that specific types of affirmations concerning the self as indicated by endorsements of the PPI items are associated with quite different patterns of activation in the DMN as inferred by quantification of brain activity. The effect size in the increase in the activity within the DMN structures was equivalent to a correlation of $\sim 0.48$ which is similar to the correlation between Machiavellian egocentric scores and inferences of DMN activity reported by Sheng et al. [12].

There are two major conclusions from this consistency of strength of association. First, psychometric tools of inferences for "common sense" and complex constructs such as "egocentricism" are replicable across experiments. Second, the often-maligned procedure of psychometric measurement for discerning magnitudes of a construct is reflected in quantitative alterations in brain function. Third, sLORETA revealed powerful differences within the DMN that are more conspicuous than the group differences in means for this psychometric tool. The two groups with the lowest vs. highest egocentric scores, $25 \%$ vs. $65 \%$ endorsement of the six items, were associated with a difference of 2000 vs. 3000 units of total activation. We found the results to be revealing with respect to the potential convergence of traditional psychological constructs with modern technologies such as sLORETA.

The second major result was the gender differences for the hemisphere in which the DMN activity was most predominant. As noted in Fig. (2), there was more activation of the left hemispheric DMN structures for females, while for the men there was more activation within the right hemispheric DMN structures compared to either hemisphere for the reference structures. Because structure dictates function, we would predict that the nature of egocentrism for men and women should differ. For women, the egocentricism would be more dominated by linguistic images and processes that are usually represented in the left hemisphere. In fact one would expect that awareness or exposure to syntax and semantics would contribute significantly to the strength of this egocentricism.

On the other hand for men, with a relative right hemispheric enhancement of the DMN, the egocentricism would be dominated by its properties. There would be less self-awareness of the etiologies for the egocentric references. They would be suffused with more affective (emotional) features and spatial relationships. Considering the theoretical 
and empirical studies that suggest the right hemisphere is the primary source of the "sensed presence" or "the other" which is often attributed with mystical or religious significance $[2,19]$, one would expect that male egocentric themes would be more frequently dominated by convictions of "cosmic" consent or "selection".

\section{CONFLICT OF INTEREST}

The authors confirm that this article content has no conflict of interest.

\section{ACKNOWLEDGEMENTS}

The technical contribution and advice of Professor Kevin Saroka is appreciated. Special thanks to Viger M. Persinger for her technical skills.

\section{REFERENCES}

[1] Fingelkurts AA. Persistent operational synchrony within brain default-mode network and self-processing operations in healthy subjects. Brain Cog 2011; 75: 79-90.

[2] Saroka KS, Persinger MA. Potential production of Hughlings Jackson's "parasitic consciousness" by physiologically-patterned weak transcerebral magnetic fields: QEEG and source localization. Epilep Behav 2013; 28: 395-407.

[3] Petrides M. Lateral frontal cortical contribution to memory. Sem Neurosci 1996; 8: 57-63.

[4] Schnider A. Spontaneous confabulation, reality monitoring and the limbic system-a review. Brain Res Rev 2001; 36: 150-60.

[5] Bartels A, Zeki S. The neural basis of romantic love. NeuroReport 2000; 11(17): 3829-34.

[6] Krill A, Platek SM. In-group and out-group membership mediates anterior cingulated activation to social exclusion. Front Evolution Neurosci 2009; 1:1.
[7] Kringelbach ML, Berridge KC. The functional neuroanatomy of pleasure and happiness. Discov Med 2010; 9(49): 579-87.

[8] Ino T, Nakai R, Azuma T, Kimura T, Fukuyama H. Brain activation during autobiographical memory retrieval with special reference to the default mode network. Open Neuroimag J 2011; 5: 14-23.

[9] Buckner RL, Petersen SE. What does neuroimaging tell us about the role of the prefrontal cortex in memory retrievel? Semin Neurosci 1996; 8: 47-55.

[10] Sheline YI, Barch DM, Price JL, et al. The default mode network and self-referential processes in depression. PNAS 2008; 106(6): 1942-7.

[11] Irish M, Piguet O, Hodges JR. Self-projection and the default network in frontotemporal dementia. Nat Rev Neurol 2012; 14(8): 152-6.

[12] Sheng T, Gheytanchi A, Aziz-Zadeh L. Default network activations are correlated with psychopathic personality traits. PLoS ONE 2010; 5(9): e12611.

[13] Persinger MA, Makarec K. Complex partial epileptic-like signs as a continnum from normals to epileptics: normative data and clinical populations. J Clin Psychol 1983; 49: 33-45.

[14] Persinger MA, Makarec K. Temporal lobe signs and correlative behaviors displayed by normal populations. J Gen Psychol 1986; 114: 179-95.

[15] Persinger MA, Makarec K. Interactions between temporal lobe signs, imaginings, beliefs, and gender: the effect upon logical inference. Imagin Cogn Pers 1991; 11: 149-66.

[16] Persinger MA, Makarec K. The feeling of a presence and verbal meaningfulness in context of temporal lobe function: factor analytic verification of the Muses? Brain Cogn 1992; 43: 1081-6.

[17] Makarec K, Persinger MA. Electroencephalographic validation of a temporal lobe signs inventory in a normal population. J Res Pers 1990; 24: 323-7.

[18] Pascual-Marqui RD, Esslen M, Kochi K, Lehmann D. Functional imaging with low-resolution brain electromagnetic tomography (LORETA): a review. Method Find Exp Clin 2002; 24: 91-5.

[19] Persinger MA. The sensed presence within experimental settings: implications for the male and female concept of self. J Psychol 2003; 137: 5-16.

(C) Collins and Persinger; Licensee Bentham Open.

This is an open access article licensed under the terms of the Creative Commons Attribution Non-Commercial License (http://creativecommons.org/licenses/by$\mathrm{nc} / 3.0 /$ ), which permits unrestricted, non-commercial use, distribution and reproduction in any medium, provided the work is properly cited. 